\title{
Role of interluekin-6 and insulin resistance as screening markers for metabolic syndrome in patients of chronic obstructive pulmonary disease. A hospital based cross-sectional study
}

\author{
Manu Dogra $^{1}$, Surabhi Jaggi ${ }^{1}$, Deepak Aggarwal ${ }^{1}$, Seema Gupta ${ }^{2}$, Varinder Saini ${ }^{1}$, Jasbinder Kaur ${ }^{2}$ \\ ${ }^{1}$ Department of Pulmonary Medicine; ${ }^{2}$ Department of Biochemistry, Government Medical College and Hospital, \\ Chandigarh, India
}

\begin{abstract}
Chronic obstructive pulmonary disease (COPD) is usually associated with a variety of extra-pulmonary manifestations. Metabolic syndrome (MetS) is one such entity that has been scarcely studied in Indian patients. Availability of a good screening marker may help in timely detection of this co morbidity in COPD
\end{abstract}

Correspondence: Dr Deepak Aggarwal, Professor, Department of Pulmonary Medicine, Government Medical College and Hospital, sector 32, Chandigarh 160030, India.

Mobile: +91.9646121584 .

E-mail: drdeepak@hotmail.com

Key words: HOMA-IR; insulin resistance syndrome; cross-sectional study.

Contributions: All the authors made a substantive intellectual contribution, have read and approved the final version of the manuscript and agreed to be accountable for all aspects of the work.

Conflict of interest: The authors declare that they have no competing interests, and all authors confirm accuracy.

Ethics approval: This study was approved by the Institutional Ethics Committee of Government Medical College and Hospital, Chandigarh, India (No. GMC/TA-I/(19-D)/2014/43649 dated 08.12.2014).

Informed consent: Written informed consent was taken from all the study participants.

Received for publication: 21 July 2021.

Accepted for publication: 20 December 2021.

Publisher's note: All claims expressed in this article are solely those of the authors and do not necessarily represent those of their affiliated organizations, or those of the publisher, the editors and the reviewers. Any product that may be evaluated in this article or claim that may be made by its manufacturer is not guaranteed or endorsed by the publisher.

${ }^{\circ}$ Copyright: the Author(s), 2021

Licensee PAGEPress, Italy

Monaldi Archives for Chest Disease 2022; 92:2024

doi: 10.4081/monaldi.2021.2024

This article is distributed under the terms of the Creative Commons Attribution-NonCommercial International License (CC BY-NC 4.0) which permits any noncommercial use, distribution, and reproduction in any medium, provided the original author(s) and source are credited. patients. We conducted a cross sectional study to evaluate the prevalence of MetS among COPD patients and to evaluate the role of Interleukin-6 and insulin resistance (as measured by HOMA-IR) as screening markers for MetS in COPD. A total of 100 stable COPD patients were evaluated for MetS using US National Cholesterol Education Program Adult Treatment Panel III (2005) guidelines. Interleukin-6 and HOMA-IR (for insulin resistance) were measured and compared between COPD patients with and without MetS. ROC analysis was done to find the best cut-off value and sensitivity and specificity of both the molecules in detecting MetS. In the results, the mean age of the study cohort was $59.9 \pm 8.7$ years (males=93). Forty five COPD patients $(45 \%)$ fulfilled the criteria for MetS. Patients with MetS were comparatively younger (57.9 \pm 9.5 vs $61.6 \pm 7.8$ years; $\mathrm{p}=0.037)$ but had longer duration of preceding COPD (9.9 \pm 2.8 vs $6.0 \pm 2.2$ years; $\mathrm{p}<0.001)$ as compared to those without MetS. Both IL-6 and HOMA index were statistically higher $(\mathrm{p}<0.05)$ in COPD-MetS patients as compared to the other group. At cutoff value of $36.3 \mathrm{pg} / \mathrm{ml}$ for IL-6 and 1.61 for HOMA index, IL-6 and HOMA-IR had sensitivity $91.1 \%$ and $82.2 \%$ respectively in detecting MetS among COPD patients. To conclude, metabolic syndrome is a common comorbidity seen in COPD patients. Interleukin-6 has a better sensitivity than HOMAIR in screening MetS among COPD patients.

\section{Introduction}

Chronic obstructive pulmonary disease (COPD) is a debilitating airway disease affecting $4-5 \%$ of the Indian population [1]. According to World health organization, it is the third leading cause of disease related death worldwide [2]. Current evidence labels it as a systemic disease with a number of associated extrapulmonary/systemic manifestations, notably, osteoporosis, diabetes mellitus (DM), cardiovascular diseases and depression [3].

Metabolic syndrome (MetS), also called insulin resistance syndrome or syndrome $\mathrm{X}$ is a constellation of metabolic risk factors namely insulin resistance, abdominal obesity, elevated blood pressure and lipid abnormalities (elevated level of triglycerides and low level of high-density lipoprotein (HDL) cholesterol) that increases the risk of developing cardiovascular disease and DM. This syndrome has been reported in COPD patients, in the range of $21-58 \%$ in different studies [4-9]. There is scarcity of data from India with two previous studies projecting the prevalence at $27.8 \%$ [5] and 54\% [10].

Occurrence of MetS in COPD has been attributed to certain common pathogenetic mechanisms/factors like systemic inflammation, adipose tissue inflammation, physical inactivity, smoking and 
genetics $[11,12]$. Role of systemic inflammation is supported by increased levels of certain pro-inflammatory cytokines like Interleukin-6 (IL-6) both in COPD and MetS [13-15]. Systemic and adipose tissue inflammation associated with COPD also results insulin resistance (IR) which is considered the harbinger of MetS. Previous studies have also been found higher level of IR in COPD patients as compared to controls $[16,17]$. This might increase future risk of cardiovascular diseases and DM in COPD patients [18].

With the change in lifestyle, the prevalence of MetS is rapidly increasing in India. It is estimated that approximately $40 \%$ of north Indian population is affected by MetS [19]. Despite COPD and MetS being common in India, the data on the prevalence of MetS in COPD is scarce [5,10]. Moreover, there is an impending need for an effective screening marker that can timely detect MetS among COPD patients. Interleukin-6 and HOMA-IR are 2 promising markers that have been sparingly evaluated in COPD patients. Hence, the present study was conducted to determine the prevalence of MetS among COPD patients in this geographical region as well as to evaluate the role of IL-6 and HOMA-IR as screening markers for MetS in COPD.

\section{Materials and Methods}

This was a cross-sectional study conducted in a tertiary care hospital over a period of 2 years. Patients of stable COPD attending the pulmonary OPD, irrespective of their disease stage and duration, were consecutively enrolled. COPD was diagnosed according to GOLD guidelines [20], by the presence of persistent respiratory symptoms and airflow limitation, as reflected by postbronchodilator $\mathrm{FEV}_{1} / \mathrm{FVC}<0.70$. Clinical stability was ascertained by the absence of exacerbation in the preceding 6 weeks. Patients with concomitant i) obstructive sleep apnea; ii) lung cancer; iii) super added lower respiratory tract infection; iv) cardiac disease; or v) those having any evidence of systemic infection were excluded as they may confound the values of the markers under evaluation. Based on the results of systematic review [4], a sample size of 87 was required to detect $34 \%$ prevalence of metabolic syndrome in COPD with $95 \%$ confidence level and 10\% permissible error (OpenEpi, v. 3). After adjusting for drop outs/incomplete data, it was decided to enroll 100 COPD patients. Informed written consent was taken from all the subjects. The study was approved by the Institutional Ethics Committee of the Hospital.

\section{Methods}

All patients were subjected to detailed history and clinical examination highlighting their demographics, duration of COPD and smoking status. They were subjected to routine spirometry according to standard guidelines [21]. The test was performed using spirometer make Spiro Analyser, model no. ST-90 (Fakuda Sangyo Co. Ltd, Tokyo, Japan) and their post bronchodilator $\mathrm{FEV}_{1}, \mathrm{FVC}$ and $\mathrm{FEV}_{1} / \mathrm{FVC}$ values were measured. The severity of airflow limitation was graded into 4 GOLD stages (1-4) based on recent GOLD guidelines [20].

All subjects were asked to come fasting on subsequent day when they were evaluated for the presence of MetS using US National Cholesterol Education Program Adult Treatment Panel III (2005) guidelines [22]. As per these guidelines, the diagnosis of MetS require the presence of $\geq 3$ criteria out of a total of 5 . These criteria include fasting blood sugar, waist circumference, blood pressure, high density lipoprotein and triglycerides. Waist circumference was measured at the midpoint between lower costal margin and superior iliac crest in the mid axillary line. Blood pressure was measured twice using sphygmomanometer in a seated position after 10 minutes resting. Two readings of systolic and diastolic blood pressure were recorded in 5 minutes interval and the average was used for data analysis. Fasting blood glucose, triglycerides and HDL cholesterol were measured in $10 \mathrm{ml}$ of fasting venous blood sample by standard method on Random Access Chemistry Analyzer modular P-800. Based on the values and the number of criteria fulfilled, patients were diagnosed to have MetS.

Thereafter, sample for insulin and IL-6 levels were collected from all subjects in citrate vials. Insulin resistance was measured by HOMA-IR, that was calculated using the following equation: (fasting insulin $(\mu \mathrm{U} / \mathrm{ml}) \mathrm{X}$ fasting glucose $(\mathrm{mmol} / \mathrm{l})) / 22.5$

Subsequently, IL-6 levels and HOMA-IR values were compared in COPD patients with and without MetS.

\section{Statistical analysis}

Quantitative data was summarized as mean \pm SD or median (interquartile range), as appropriate, and categorical variables were presented as $\mathrm{n}(\%)$. Comparison of quantitative and categorical variables between the COPD patients with and without metabolic syndrome were done using student $\mathrm{T}$ test/Mann-Whitney test and Chi square test/Fischer exact test, respectively. Spearman correlation coefficient was used to find correlation between IL-6 and HOMA-IR. Multiple logistic regression analysis was performed using forward LR approach and odds ratio (OR) (with 95\% confidence interval) was calculated to find association between different variables and MetS in the COPD patients. Receiver operating characteristic (ROC) curves were calculated to find maximal cutoff values of IL-6 and HOMA-IR for detecting MetS and sensitivity and specificity were calculated for those cut-off values among the patients. All statistical tests were two-sided with $\mathrm{p}<0.05$ taken as statistically significant. All statistical calculations were done using computer program SPSS (IBM SPSS Statistics 21.0; Armonk, NY, USA).

\section{Results}

COPD patients mainly comprised of elderly (mean age $59.9 \pm 8.7$ years) males ( $\mathrm{n}=93$ ). Mean duration of COPD symptoms was $7.8 \pm 3.2$ years. Mean post bronchodilator $\mathrm{FEV}_{1} \%$ was $48.9 \pm 19.3$ (Table 1). Thirty-eight patients presented in moderate stage $(n=38)$ of the disease followed by 32 and 22 in severe and very severe stage, respectively.

\section{Prevalence of metabolic syndrome in COPD patients}

Forty five patients out of the study cohort (45\%) fulfilled the criteria for MetS. Out of the 5 components of MetS, elevated blood pressure was the most common $(\mathrm{n}=38)$ followed by elevated fasting blood glucose $(n=37)$, elevated triglycerides $(n=34)$, reduced HDL $(n=27)$ and elevated waist circumference $(n=7)$. Patients with MetS were relatively younger $(57.9 \pm 9.5 v s 61.6 \pm 7.8$ years; $\mathrm{p}=0.037)$, had poorer lung function $\left(\mathrm{FEV}_{1} \% 44.9 \pm 17.2\right.$ vs $\left.52.3 \pm 20.5 ; \mathrm{p}=0.05\right)$ and longer duration of COPD $(9.9 \pm 2.8 v s 6.0 \pm 2.2$ years; $\mathrm{p}<0.001)$ than the other group (Table 1). However, there was no significant difference in the distribution of GOLD stage between the 2 groups $(\mathrm{p}=0.10)$ (Figure 1). Number of patients with diabetes was higher in the COPD-MetS group $(\mathrm{n}=19 ; 42.2 \%)$ as compared to COPD without MetS $(n=3 ; 0.05 \%)(\mathrm{p}<0.001)$.

On multivariate logistic regression analysis, patient age (adjust- 
ed OR: $0.89 ; 95 \%$ CI: $0.83-0.96 ; \mathrm{p}=0.002)$ and duration of COPD (adjusted OR: 2.05; 95\% CI:1.5-2.7; $<<0.001$ ) were the independent factors that predicted MetS in COPD patients (Table 2).

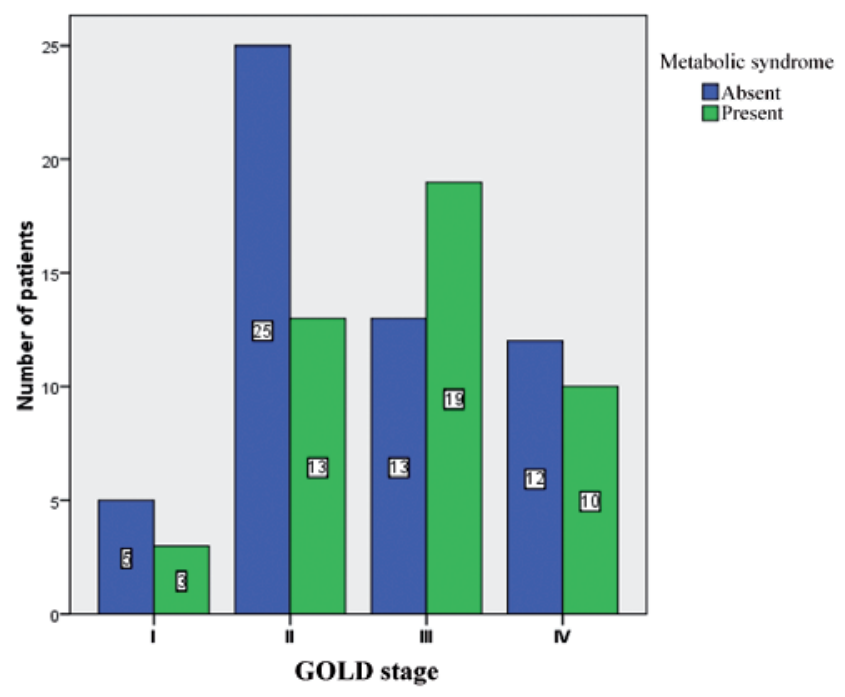

Figure 1. Bar graph showing the distribution of patients with MetS in different COPD GOLD stages.

\section{Relation of IL-6 and HOMA-IR levels with MetS in COPD patients}

Both IL-6 and HOMA-IR levels were higher in COPD patients with MetS as compared to the other group $(\mathrm{p}<0.001)$ (Table 3$)$. There was a significant correlation between IL-6 and HOMA-IR levels (Pearson $\mathrm{r}=0.51 ; \mathrm{p}<0.001$ ). On subgroup analysis, IL-6 levels were also significantly raised in COPD-MetS as compared COPD-non-MetS in both diabetic (MetS 67.8 \pm 27.5 vs non-MetS $16.8 \pm 13.1 ; \mathrm{p}<0.001)$ and non-diabetic (MetS $59.5 \pm 30.5$ vs nonMetS 24.4 $\pm 20.3 ; \mathrm{p}=0.004$ ) patients.

On ROC curve analysis, IL-6 showed a better performance than HOMA-IR in detecting metabolic syndrome (area under curve $89 \%$ vs $78 \%$ ). At cut off value of $36.3 \mathrm{pg} / \mathrm{ml}$ for IL-6 and 1.61 for HOMA-IR, IL-6 was found to have a better sensitivity than HOMA-IR (91.1\% vs 82.2\%) in detecting MetS in COPD patients (Figures 2 and 3 ).

\section{Discussion}

The present study showed a high prevalence (45\%) of MetS in COPD patients. Both IL-6 and HOMA-IR were significantly higher in COPD patients with MetS; however, IL-6 seemed to have a better sensitivity $(91.1 \%)$ than HOMA-IR $(82.2 \%)$ in detecting MetS among COPD patients.

Table 1. Comparison of parameters between COPD patients with and without MetS.

\begin{tabular}{|c|c|c|c|c|}
\hline Parameters & All COPD patients $(n=100)$ & $\begin{array}{l}\text { Metabo) } \\
\text { Present }(\mathrm{n}=45)\end{array}$ & Absent $(n=55)$ & p-value \\
\hline Age (years) & $59.9 \pm 8.7$ & $57.9 \pm 9.5$ & $61.6 \pm 7.8$ & 0.037 \\
\hline Males (n) & 93 & 41 & 52 & 0.69 \\
\hline BMI $\left(\mathrm{kg} / \mathrm{m}^{2}\right)$ & $24.3 \pm 5.5$ & $23.7 \pm 5.5$ & $24.8 \pm 5.6$ & 0.33 \\
\hline Smokers (n) & 95 & 44 & 51 & 0.37 \\
\hline Pack years & $21.2 \pm 13.2$ & $21.3 \pm 11.7$ & $21.2 \pm 14.3$ & 0.98 \\
\hline Alcohol (n) & 72 & 33 & 39 & 0.82 \\
\hline $\mathrm{FEV}_{1}(\%$ predicted $)$ & $48.9 \pm 19.3$ & $44.9 \pm 17.2$ & $52.3 \pm 20.5$ & 0.057 \\
\hline Duration of COPD symptoms (years) & $7.8 \pm 3.2$ & $9.9 \pm 2.8$ & $6.0 \pm 2.2$ & $<0.001$ \\
\hline
\end{tabular}

BMI, body mass index; $\mathrm{FEV}_{1}$, forced expiratory volume in $1^{\mathrm{st}}$ second. All values are mentioned a mean $\pm \mathrm{SD}$ or $\mathrm{n}$.

Table 2. Logistic regression analysis to evaluate association between metabolic syndrome and different patient parameters.

\begin{tabular}{lccc} 
Parameter & $\begin{array}{c}\text { Univariate analysis } \\
\text { Odds ratio }(95 \% \mathrm{CI})\end{array}$ & $\begin{array}{c}\text { Multivariate analysis } \\
\text { p-value }\end{array}$ & $\begin{array}{c}\text { Adjusted odds ratio (95\% CI) } \\
\text { Age }\end{array}$ \\
Males & $0.95(0.91-0.99)$ & 0.04 & $0.89(0.83-0.96)$ \\
\hline Pack years & $0.59(0.13-2.8)$ & 0.50 & 0.002 \\
FEV $\%$ & $0.99(0.97-1.02)$ & 0.06 & \\
\hline Duration of symptoms & $0.98(0.96-1.001)$ & $<0.001$ & $2.05(1.5-2.7)$
\end{tabular}

Table 3. Comparison of IL-6 and Homa-IR levels in COPD patients with and without MetS.

\begin{tabular}{lccc} 
Parameter & COPD with MetS & COPD without MetS & P-value \\
IL-6 $(\mathrm{pg} / \mathrm{ml})$ & $66.5 \pm 27.8$ & $22.8 \pm 20.7$ & $<0.001$ \\
Homa-IR & $4.5 \pm 2.8$ & $1.9 \pm 1.8$ & $<0.001$ \\
\hline
\end{tabular}


The prevalence of MetS in COPD has been highly variable in previous studies, with figures ranging between $21-58 \%$ [4-9]. This non-uniformity in the prevalence figures is not only due to difference in the study designs and the diagnostic criteria used for MetS, but also reflect multifactorial dependence of MetS. Hypertension was the most prevalent MetS component seen in the present study that was similar to previous studies $[4,23]$. The present study evaluated common patient and disease related factors that might predispose COPD patients to MetS. The results showed that age was an independent factor that predicted MetS in COPD in a logistic regression model (adjusted $\mathrm{OR}=0.89$; 95\% CI-0.83-0.96; $\mathrm{p}=0.002$ ). This is in contrast to a Polish study in which age was statistically similar in the 2 groups [7]. However, another study demonstrated a higher prevalence of MetS in younger patients with less severe COPD and defined it as a separate phenotype of COPD [24]. Apart from age, duration of COPD symptoms was another factor that was associated with MetS (adjusted OR=2.05; 95\% CI$1.5-2.7 ; \mathrm{p}<0.001)$. The above findings imply that the patients who develop COPD at a younger age were more likely to develop MetS and the likelihood increased with the duration of COPD.

Association between presence of metabolic syndrome and lung function in COPD is an area that lacks clarity in previous studies. One evidence suggests a relatively high prevalence of MetS in patients with mild to moderate disease [10,25-27]. This is attributed to presence of cachexia and wasting in severe and very severe COPD patients that apparently lowers the incidence of MetS in later stages. However, few other studies have failed to prove any association between MetS and the COPD stage [6,7] that was also seen in the present study (Figure 1). A systematic review established that MetS was more prevalent in overweight and obese female patients [4]. In contrast, no such association was seen in the present study. Apart from obesity, there was no difference in smoking addiction between the COPD patients with and without MetS, that was similar to previous studies $[7,10]$. However, a low percentage of non smokers $(5 \%)$ among the COPD patients might have affected the results. A French study showed that current smokers are more prone to develop MetS primarily through systemic inflammatory response [28]. Moreover, a positive association of smoking with hypertension [29] and diabetes [30] also validates its role in predisposing MetS in COPD patients.

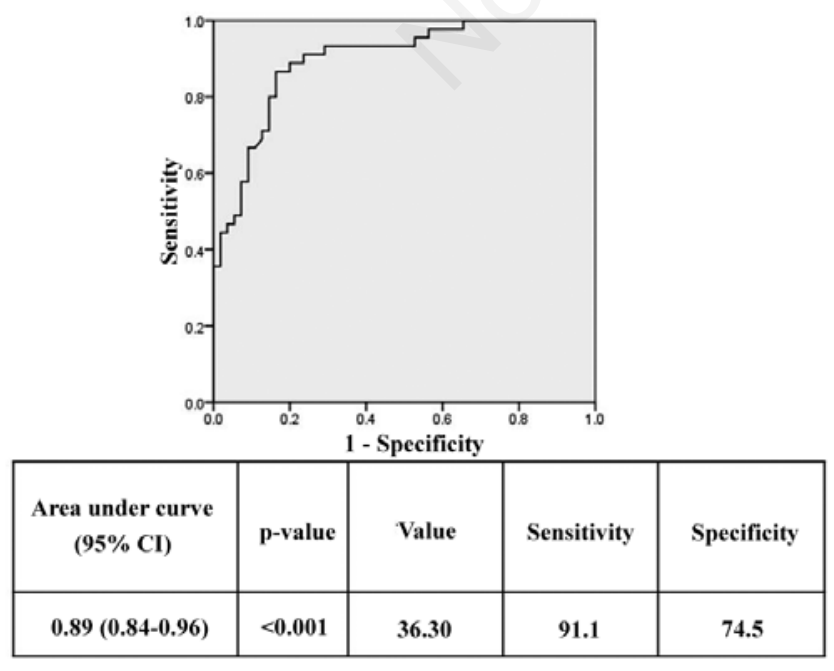

Figure 2. ROC curve, sensitivity and specificity of IL-6 for the detection of metabolic syndrome in COPD patients.
The present study evaluated the role of Interleukin-6, a proinflammatory cytokine, as a screening marker for MetS in COPD patients. The results showed significantly high levels of IL-6 in COPD-MetS patients as compared COPD-non-MetS $(\mathrm{p}<0.001)$, with a sensitivity of $91.1 \%$ in detecting MetS. The results were further validated on sub-group analysis in which IL-6 levels were also elevated in non-diabetic COPD patients with MetS (COPD-MetS $59.5 \pm 30.5$ vs non-MetS $24.4 \pm 20.3 \mathrm{pg} / \mathrm{ml} ; \mathrm{p}=0.004$ ). Moreover, the lack of correlation between IL-6 levels and pack years of smoking (Pearson $\mathrm{r}=0.10 ; \mathrm{p}=0.29$ ) also negated the confounding effect of smoking on IL-6 levels. The results are in coherence with a previous study that also showed a statistically significant correlation between IL-6 levels and incidence of metabolic syndrome $(p=0.021)$ [13]. Apart from IL-6, other markers of systemic inflammation like C-reactive protein and fibrinogen have also been studied for their potential association with COPD and MetS [31-33]. However, IL-6 was selected for evaluation in the study as it is a primary cytokine regulator of CRP production, fibrinogen and thrombocytosis that confirmed its suitability for evaluation as a potential screening marker [31,34].

Interestingly, there was no correlation between the IL-6 levels and the $\mathrm{FEV}_{1}$ values in the current study (Pearson $\mathrm{r}=-0.13$; $\mathrm{p}=0.21$ ) that was similar to the previous study [13]. However, data from the Framingham Heart Study showed a significant negative correlation between IL-6 levels and $\mathrm{FEV}_{1}$ values [35]. Further research with large sample size and adjusting for confounders might help to clarify the relationship.

Insulin resistance (IR) is an independent risk factor for MetS and its cardiovascular complications. It is postulated that poor oxygenation in COPD leads to adipose tissue hypoxia and inflammation, lipid dysregulation and hypoadiponectinemia that adversely affects insulin signaling. The cascades of events lead to hyperinsulinemia that causes endothelial dysfunction leading to hypertension and further insulin resistance. Previous studies have demonstrated increase in IR in COPD-MetS as compared to those without MetS $[24,36]$. The present study also validated the previous findings (HOMA-IR in COPD-MetS $4.5 \pm 2.8$ vs COPD 1.9 \pm 1.8 ; $\mathrm{p}=0.001)$. On ROC curve analysis, IL-6 showed a better performance in detecting MetS as compared to HOMA-IR (area under curve - interleukin-6 89\% vs HOMA-IR 78\%) in the study. At cut-

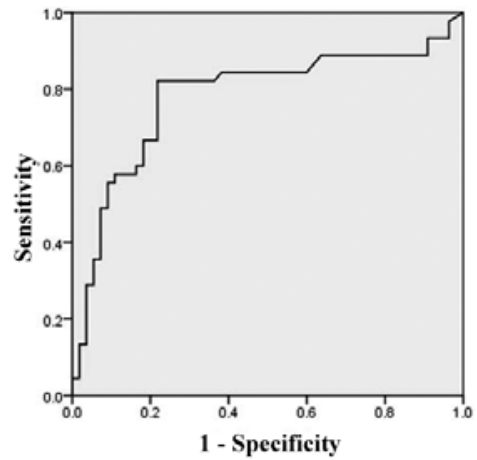

\begin{tabular}{|c|c|c|c|c|}
\hline $\begin{array}{c}\text { Area under curve } \\
(95 \% \mathrm{CI})\end{array}$ & p-value & Value & Sensitivity & Specificity \\
\hline $0.78(0.68-.088)$ & $<0.001$ & 1.61 & 82.2 & 74.5 \\
\hline
\end{tabular}

Figure 3. ROC curve, sensitivity and specificity of HOMA-IR for the detection of metabolic syndrome in COPD patients. 
off values of 36.3 for IL- 6 and 1.61 for HOMA-IR, IL- 6 had a better sensitivity than HOMA-IR in detecting MetS $(99.1 \%$ vs $82.2 \%)$. Both markers also showed a moderately positive correlation with each other (Pearson $r=0.51 ; p<0.001$ ) that was also demonstrated in a previous study $(r=0.276, p=0.039)$ [18]. The above results reaffirm the role of common/interlinked inflammatory pathway in the pathogenesis of MetS.

The present study is one of the first studies from this geographical region to give a comprehensive picture on the prevalence of MetS in COPD patients as well as the role of IL-6 and HOMA-IR in screening MetS. However, the cross sectional design limited its ability to evaluate dynamic changes in the markers with time. Future studies using longitudinal design may help to further add to the evidence.

To conclude, COPD patients are frequently associated with MetS. The combined COPD-MetS seems to be a specific phenotype that is common in younger patients with early onset of COPD. With a higher sensitivity and equal specificity, IL-6 seems to be a better screening marker than HOMA-IR in detecting MetS in these patients. The results suggest that all COPD patients, particularly young and those with early disease onset should be screened using these biomarkers for early detection and treatment of MetS.

\section{References}

1. McKay AJ, Mahesh PA, Fordham JZ, Majeed A. Prevalence of COPD in India: a systematic review. Prim Care Respir J 2012; 21: 313-21.

2. World Health Organization. The top 10 causes of death. Available from: https://www.who.int/news-room/factsheets/detail/the-top-10-causes-of-death

3. Mannino DM, Thorn D, Swensen A, Holguin F. Prevalence and outcomes of diabetes, hypertension and cardiovascular disease in COPD. Eur Respir J 2008;32:962-9.

4. Cebron Lipovec N, Beijers RJ, van den Borst B, et al. The prevalence of metabolic syndrome in chronic obstructive pulmonary disease: A systematic review. COPD 2016;13:399-406.

5. Sridharan VSHK, Acharya V. Comorbidities of chronic obstructive pulmonary disease and their affect on hospitalization of patients in a tertiary care hospital. J Community Hosp Intern Med Perspect 2021;11:120-23

6. Breyer MK, Spruit MA, Hanson CK, et al. Prevalence of metabolic syndrome in COPD patients and its consequences. PloS One 2014;9:e98013.

7. Rubinsztajn R, Przybylowski T, Maskey-Warzechowska M, et al. Metabolic syndrome as a factor affecting systemic inflammation in patients with chronic obstructive pulmonary disease. Adv Exp Med Biol 2017;1021 55-62.

8. Singh NK, Karki L. Metabolic syndrome in patients with chronic obstructive pulmonary disease in medicine department of a tertiary care hospital: A descriptive cross-sectional study. JNMA J Nepal Med Assoc 2021;59:313-16.

9. Kiani FZ, Ahmadi A. Prevalence of different comorbidities in chronic obstructive pulmonary disease among Shahrekord PERSIAN cohort study in southwest Iran. Sci Rep 2021;11:1548.

10. Priyadharshini N, Renusha RC, Reshma S, et al. Prevalence of metabolic syndrome in patients with chronic obstructive pulmonary disease: An observational study in South Indians. Diabetes Metab Syndr 2020;14:503-7.

11. Agusti A, Soriano JB. COPD as a systemic disease. COPD 2008 5:133-8.
12. Barnes PJ, Celli BR. Systemic manifestations and comorbidities of COPD. Eur Respir J 2009;33:1165-85.

13. Hosny H, Abdel-Hafiz H, Moussa H, Soliman A. Metabolic syndrome and systemic inflammation in patients with chronic obstructive pulmonary disease. Egypt J Chest Dis Tuberc 2013; 62: 85-9.

14. Garcia-Rio F, Miravitlles M, Soriano JB, et al. Systemic inflammation in chronic obstructive pulmonary disease: a population-based study. Respir Res 2010;11 63.

15. Wei J, Xiong XF, Lin YH, et al. Association between serum interleukin-6 concentrations and chronic obstructive pulmonary disease: a systematic review and meta-analysis. Peer J 2015;3:e1199.

16. Kiran Z, Majeed N, Zuberi BF. Comparison of frequency of insulin resistance in patients with chronic obstructive pulmonary disease with normal controls. Pak J Med Sci 2015;31:1506-10.

17. Eker S, Ayaz L, Tamer L, Ulubas B. Leptin, visfatin, insulin resistance, and body composition change in chronic obstructive pulmonary disease. Scand J Clin Lab Invest 2010; 70:40-4.

18. Bolton CE, Evans M, Ionescu AA, et al. Insulin resistance and inflammation - A further systemic complication of COPD. COPD 2007;4:121-6.

19. Khan Y, Lalchandani A, Gupta AC, et al. Prevalence of metabolic syndrome crossing $40 \%$ in Northern India: Time to act fast before it runs out of proportions. J Family Med Prim Care 2018;7:118-23.

20. Global Initiative for Chronic Obstructive Lung Disease (GOLD). Global strategy for the diagnosis, management and prevention of COPD (updated 2016). Accessed: Oct 2, 2016. Available from: https://goldcoped.org/

21. Miller MR, Hankinson J, Brusasco V, et al. Standardisation of spirometry. Eur Respir J 2005;26:319-38.

22. Grundy SM, Cleeman JI, Daniels SR, et al. Diagnosis and management of the metabolic syndrome: an American Heart Association/National Heart, Lung, and Blood Institute scientific statement: Executive Summary. Crit Pathw Cardiol 2005;4:198-203.

23. Choi HS, Rhee CK, Park YB, et al. Metabolic syndrome in early chronic obstructive pulmonary disease: Gender differences and impact on exacerbation and medical costs. Int J Chron Obstruct Pulmon Dis 2019;14:2873-83.

24. Minas M, Kostikas K, Papaioannou AI, et al. The association of metabolic syndrome with adipose tissue hormones and insulin resistance in patients with COPD without co-morbidities. COPD 2011;8:414-20.

25. Koul PA. Metabolic syndrome and chronic obstructive pulmonary disease. Lung India 2016;33:359-61.

26. Piazzolla G, Castrovilli A, Liotino V, et al. Metabolic syndrome and chronic obstructive pulmonary disease (COPD): The interplay among smoking, insulin resistance and vitamin D. PloS One 2017;12 e0186708.

27. Watz H, Waschki B, Kirsten A, et al. The metabolic syndrome in patients with chronic bronchitis and COPD: frequency and associated consequences for systemic inflammation and physical inactivity. Chest 2009;136:1039-46.

28. Geslain-Biquez C, Vol S, Tichet J, et al. The metabolic syndrome in smokers. The D.E.S.I.R. study. Diabetes Metab 2003;29:226-34.

29. Elliott JM, Simpson FO. Cigarettes and accelerated hypertension. N Z Med J 1980;91:447-9.

30. Rimm EB, Chan J, Stampfer MJ, Colditz GA, Willett WC. 
Prospective study of cigarette smoking, alcohol use, and the risk of diabetes in men. BMJ 1995;310:555-9.

31. Gan WQ, Man SF, Senthilselvan A, Sin DD. Association between chronic obstructive pulmonary disease and systemic inflammation: a systematic review and a meta-analysis. Thorax 2004;59:574-80.

32. Ropcke S, Holz O, Lauer G, et al. Repeatability of and relationship between potential COPD biomarkers in bronchoalveolar lavage, bronchial biopsies, serum, and induced sputum. PloS One 2012;7:e46207.
33. Naik D, Joshi A, Paul TV, Thomas N. Chronic obstructive pulmonary disease and the metabolic syndrome: Consequences of a dual threat. Indian J Endocrinol Metab 2014;18:608-16.

34. Kishimoto T. Interleukin-6: from basic science to medicine--40 years in immunology. Annu Rev Immunol 2005;23:1-21.

35. Walter RE, Wilk JB, Larson MG, et al. Systemic inflammation and COPD: the Framingham heart study. Chest 2008;133:19-25.

36. Diez-Manglano J, Barquero-Romero J, Almagro P, et al. COPD patients with and without metabolic syndrome: clinical and functional differences. Intern Emerg Med 2014;9:419-25. 